\title{
NOTE ON THE FLUORESCENCE OF FROZEN SOLUTIONS OF THE URANYL SALTS.
}

\author{
By Edward L. Nichols and Ernest Merritt.
}

THAT aqueous solutions of the uranyl salts exhibit fluorescence similar to that of the salts themselves but of a feebler intensity has long been known. To determine whether the nearly equidistant bands, of which in general the fluorescence spectra of these solutions consist, retain their character when the solutions are frozen and reduced to the temperature of liquid air or whether resolution into groups of narrower bands occurs, was the purpose of the experiments to be described in this preliminary note.

To this end solutions of uranyl sulphate, uranyl potassium sulphate, uranyl nitrate and uranyl acetate of widely varying concentrations were used.

The solutions were placed in a test-tube $F$ (Fig. I) within a cylindrical

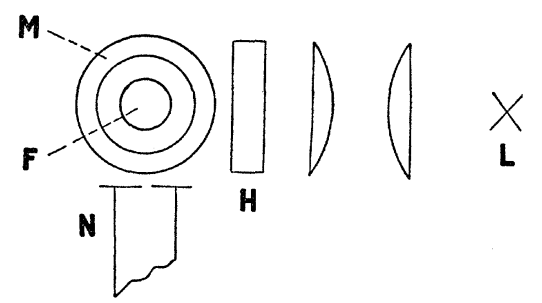

Fig. 1.

Dewar flask $M$ with unsilvered walls. Fluorescence was excited by a conical beam from a right-angled carbon arc $L$ and the spectrum was observed with a Hilger spectroscope, the collimator of which is shown at $N$. For this spectroscope a suitable spectrograph could be substituted when photographs were desired.

A ray filter $H$ absorbed all light from about wave-length $.47 \mu$ to the extreme red so that the fluorescence bands appeared upon a dark background.

In no case, with the exception of uranyl acetate in alcohol, was a resolution of the fluorescence of aqueous solutions into narrow line-like bands observable; but there were certain striking changes in the character of 
the spectra when temperature and concentration were varied. These are noted briefly in the following paragraphs:

\section{A. Aqueous Solutions.}

I. Uranyl Sulphate.-The solutions of this salt present the simplest case; that of a single set of rather broad bands essentially unmodified either by temperature or dilution excepting as to breadth and intensity. In this preliminary survey a set of solutions were made by diluting the concentrated solution successively with ten parts of water. Measurements were made of the stronger bands at the temperature of liquid air to determine approximately the location of the crests.

The results are given in Table I. In this table the bands measured are designated arbitrarily by letters $c, d, e, f$, and $g$; there being two bands, $a$ and $b$, of longer wave-length discernible in the spectroscope which do not appear in the photographs.

TABLE I.

Uranyl Sulphate in Water $-185^{\circ} \mathrm{C}$.

\begin{tabular}{c|c|c|c|c|c}
\hline \hline Concentration. & Band $c$. & Band $d$. & Band $e$. & Band $f$. & Band $g$. \\
\hline 1.00 & $.5631 \mu$ & $.5380 \mu$ & $.5131 \mu$ & $.4915 \mu$ & $.4807 \mu$ \\
0.10 & .5625 & .5377 & .5130 & .4915 & .4797 \\
0.01 & .5626 & .5380 & .5131 & .4916 & .4796 \\
0.001 & .5626 & .5376 & .5129 & .4915 & .4804 \\
0.0001 & .5632 & .5388 & .5138 & .4929 & $?$ \\
\hline
\end{tabular}

Estimations of width were also made but these are not included in the table because in a photograph the apparent width of a band depends on the intensity of the exciting light, the time of exposure and the development of the negative. As development progresses the crests appear first and darkening extends laterally outwards in both directions.

The widths as they appear in the photographs were roughly about 60 Angström units for band $c, 80$ units for bands $d, e$ and $f$ and 50 units for band $g$.

Within the errors of observation, which would appear to be one or two Angström units in the case of the more intense bands $e$ and $f$, two or three units for band $d$, three or four units for band $c$ and perhaps ten units for band $g$ which was very vague, broad and weak, there is no shift with the concentration, ascertainable by this method, until a dilution to .oor is passed. In the weakest solution (.ooor) however the bands are shifted to the red. The movement is about fourteen units for band $f$, eight or ten units for bands $d$ and $e$ and not more than five units for band $c$. The location of band $g$ for this solution was not possible on 
account of weakness of the spectrum and the disturbance due to the presence of the neighboring absorption band of liquid air.

No attempt was made to secure photographs of the spectra of still weaker solutions, but a series of visual observations made by freezing and exciting aqueous solutions each of which contained twice the proportion of water of the preceding one showed that the two brightest bands $(e$ and $f$ ) were still dimly visible when a solution containing one part of the concentrated solution to 500,000 of water was excited at the temperature of liquid air.

2. Uranyl Nitrate.-The concentrated aqueous solution of this salt when frozen and excited to fluorescence showed a double series of bright bands alternately narrow and broad. The narrower bands were on the side towards the violet, perhaps a third as wide as the accompanying broad band and less intense. The spectrum increased rapidly in brightness as cooling proceeded.

When the solution was diluted to I/IO, the fluorescence on cooling first appeared as a single very broad continuous band from red to blue but was rapidly resolved into a double series as in the spectrum of the concentrated solution.

With further diminution of concentration the double banded spectrum is observed although not so bright; at least until $\mathrm{I} / \mathrm{IOOO}$ is reached. In solution with Io,ooo parts of water however the narrower bands could no longer be distinguished.

The location of the bands, in the case of the nitrate, as shown in Table II. is somewhat less satisfactory than with the sulphate. The drift towards longer wave-lengths as the dilution increases is however not less marked. Band $g$, as in Table I., is encroached upon by the absorption

TABLE II.

Uranyl Nitrate in Water at $-185^{\circ} \mathrm{C}$.

\begin{tabular}{|c|c|c|c|c|c|c|c|c|c|c|}
\hline \multirow{2}{*}{ Conc. } & \multicolumn{2}{|c|}{ Band. } & \multicolumn{2}{|c|}{ Band. } & \multicolumn{2}{|c|}{ Band. } & \multicolumn{2}{|c|}{ Band. } & \multicolumn{2}{|c|}{ Band. } \\
\hline & $c$. & $c^{\prime}$ & $d$. & $d^{\prime \prime}$ & $e$. & $e^{\prime}$ & $f$. & $f^{\prime}$. & $g$. & $g^{\prime}$ \\
\hline $\begin{array}{l}1.00 \\
0.10 \\
0.01 \\
0.002 \\
0.001\end{array}$ & $\begin{array}{l}.5681 \mu \\
.5682\end{array}$ & $\begin{array}{l}.5596 \mu \\
.5595\end{array}$ & $\begin{array}{l}.5430 \mu \\
.5435 \\
.5432\end{array}$ & $\begin{array}{l}.5334 \mu \\
.5338 \\
.5330\end{array}$ & $\begin{array}{l}.5185 \mu \\
.5190 \\
.5196 \\
.5207 \\
.5211\end{array}$ & $\begin{array}{l}.5092 \mu \\
.5091 \\
.5089 \\
.5103 \\
.5100\end{array}$ & $\begin{array}{l}.4964 \mu \\
.4970 \\
.4970 \\
.4977 \\
.4985\end{array}$ & $\begin{array}{l}.4873 \mu \\
.4870 \\
.4875 \\
.4882\end{array}$ & $\begin{array}{l}.4802 \mu \\
.4799 \\
.4804 \\
.4803 \\
.4805\end{array}$ & $.4724 \mu$ \\
\hline
\end{tabular}

band of liquid air to such an extent that the location of the crest is open to a considerable correction and the apparent absence of drift is probably not significant. 
3. Uranyl Acetate.-The behavior of frozen solutions of uranyl acetate is much more interesting and remarkable than that of the sulphate or nitrate. In concentrated solution this salt has a fluorescence spectrum which to the eye appears to consist of a single very broad and faint band. At the temperature of liquid air this is resolved into a group of brilliant bands nearly equally spaced and in their general appearance similar to those of the sulphate but differently located (see Table III.). Upon dilution to $\mathrm{I} / \mathrm{I} 60$ the resolved spectrum consists of broad very strong bands between which, at very low temperatures, much narrower and very much fainter bands appear.

Further dilution to $\mathrm{I} / \mathrm{I} 600$ produces another marked change. The broad and narrow bands are now supplanted, as if by partial fusion, by very broad and rather feeble bands having an intermediate position. Of the two which it is possible to locate with certainty in the photograph taken of the spectrum of this solution the one of shorter wave-length is clearly double although overlapping, having broad crests at approximately $.5086 \mu$ and $.5215 \mu$. The other broad band having its middle about .549I $\mu$ cannot be even partially resolved. The weakest solution upon which observations were made had a concentration of $\mathrm{I} / \mathrm{I} 6000$. Its spectrum consisted of broad dim unresolved bands, the two strongest of which had their crests at about $.5024 \mu$ and $.5239 \mu$. The outlying bands in the neighborhood of $.48 \mu$ and $.54 \mu$ were too vague to be located in the photograph.

The bands upon which measurements were attempted are given in the following table. Whether the changes in position should be ascribed, as in the case of the other salts, to a shift with concentration can be determined only by further observations.

TABLE III.

Uranyl Acetate in Water $-185^{\circ} \mathrm{C}$.

\begin{tabular}{|c|c|c|c|c|c|c|c|c|}
\hline \multirow{2}{*}{ Concentration. } & \multicolumn{8}{|c|}{ Character of Bands. } \\
\hline & Broad. & Narrow. & Broad. & Narrow. & Broad. & Narrow. & Broad. & Narrow. \\
\hline \multirow[t]{2}{*}{$\begin{array}{l}1: 000 \\
1: 160 \\
\end{array}$} & $\begin{array}{l}.5641 \mu \\
.5640 \\
\end{array}$ & $.5533 \mu$ & $\begin{array}{l}.5398 \mu \\
.5398 \\
\end{array}$ & $.5284 \mu$ & $\begin{array}{l}.5145 \mu \\
.5152 \\
\end{array}$ & $.5048 \mu$ & $\begin{array}{l}.4936 \mu \\
.4931 \\
\end{array}$ & $.4837 \mu$ \\
\hline & & \multicolumn{2}{|c|}{ Very Broad. } & \multicolumn{3}{|c|}{ Very Broad. } & \multicolumn{2}{|c|}{ Very Broad. } \\
\hline \multicolumn{2}{|l|}{$\begin{array}{l}1: 1600 \\
1: 16000\end{array}$} & \multicolumn{2}{|c|}{$.5419 \mu$} & \multicolumn{3}{|c|}{$\begin{array}{l}.5213 \mu \\
.5239\end{array}$} & \multicolumn{2}{|c|}{$\begin{array}{l}.5086 \mu \\
.5024\end{array}$} \\
\hline
\end{tabular}

4. Uranyl Potassium Sulphate.-Only a cursory examination of this substance was made. The concentrated aqueous solution when frozen 
and excited showed broad bright bands without any trace of resolution at the temperature of liquid air. Diluted successively to I/IO, I/Ioo, $\mathrm{I} / \mathrm{IOOo}$, and I/IOooo the spectrum retained the same appearance but the bands became broader as the concentration diminished and with the higher dilutions much dimmer.

\section{B. Alcoholic Solutions.}

Some of the uranyl salts, notably the acetate and nitrate, are soluble in ethyl alcohol. The concentrated alcoholic solution of the nitrate when reduced to the temperature of liquid air has a fluorescence spectrum consisting of alternately broad and narrow bands located as shown in Table IV.

The wave-lengths of the narrow bands is approximately the same as in the case of the aqueous solution of the nitrate but the broad bands are greatly shifted towards the red. In dilute solutions the pairs of bands are replaced by single broad bands having intermediate positions.

TABLE IV.

Uranyl Nitrate in Alcohol at $-185^{\circ} \mathrm{C}$.

\begin{tabular}{l|c|c|c|c|c|c|c|c}
\hline \hline Conc. & Broad. & Narrow. & Broad. & Narrow. & Broad. & Narrow. & Broad. & Narrow. \\
\hline $1 / 1$ & .5480 & .5337 & .5214 & .5096 & .5011 & - & .4875 & .4819 \\
$1 / 50$ & .5520 & - & .5270 & - & .5034 & - & .4886 & $.4815 ?$ \\
$1 / 500$ & .5522 & - & .5271 & - & .5040 & - & .4882 & $.4821 ?$ \\
$1 / 5000$ & .5521 & - & .5273 & - & .5041 & - & - & $.4806 ?$ \\
\hline
\end{tabular}

The narrow band at .4819 was vaguely discernible at higher dilutions but the position as indicated in the table was in question. The location of the bands of the dilute solutions does not seem to be very simply related to that of the bands of the concentrated solution although it remains nearly or quite unchanged for dilutions between I/50 and I/500o. This is easily explained if we consider these bands as resulting from the merging of neighboring bands of the concentrated solution and that the relative brightness of neighboring bands in the spectrum of the concentrated solution differs as we pass from red to violet.

The spectrum of the frozen alcoholic solution of uranyl acetate was altogether different from the various spectra already considered. It consisted, both for concentrated and dilute solutions, of numerous very narrow line-like bands superimposed upon an unresolved back ground; as though in this case a portion of the dissolved acetate had been thrown out on freezing.

The sharply resolved bands of this spectrum however, although they fall 
into equally spaced series, as is the case with the spectra of the solid uranyl salts at low temperatures, do not correspond in position, nor as to interval, with the bands of the solid acetate.

In Fig. 2 the arrangement and approximate width of the various

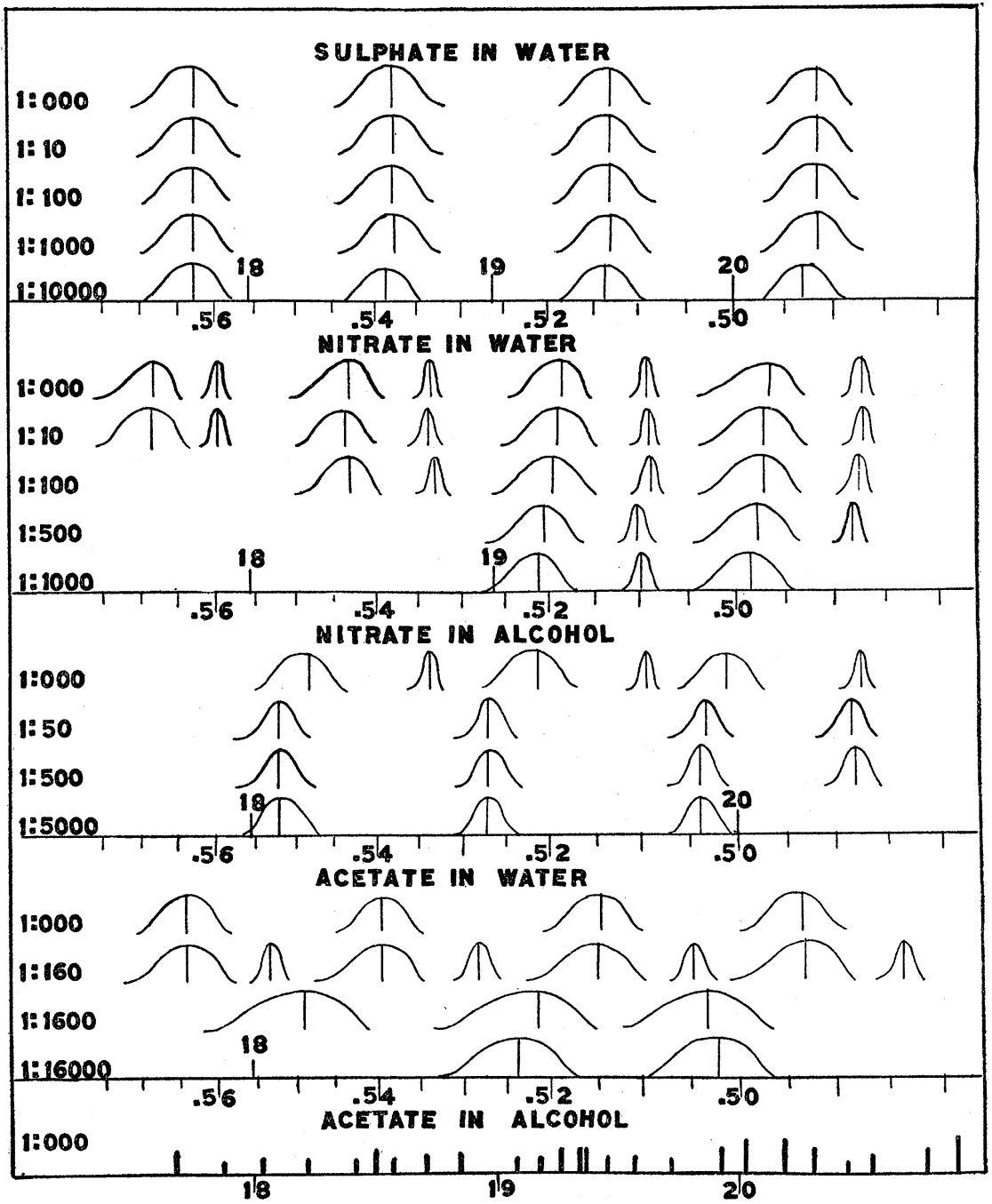

Fig. 2.

fluorescence bands already described are shown graphically in a diagram in which the abscissæ are frequencies. A scale of wave-lengths is also given for each spectrum. The positions of the narrow line-like bands in the spectrum of the acetate in alcohol are indicated at the bottom of the figure. 
It will be seen that the frequency-interval between neighboring broad bands and also between narrow bands is approximately the same in all of these spectra; the average interval being about $86 \times \mathrm{IO}^{-4}$ where the wave-length is expressed in Ångström units. The only serious exception is in the case of the dilute solution of uranyl acetate in water ( $1: 1600$ ) where very broad bands have replaced the doublets of the more concentrated solution ( $\mathrm{I}: \mathrm{I} 60$ ).

In the course of the observations recorded in this note, equally striking changes and shifts were found to occur when the temperature of a given solution was varied. A description of these phenomena may be expected as the result of a systematic study now being made by Mr. H. L. Howes, who has assisted the authors throughout this preliminary survey of the subject.

Physical Laboratory, Cornell University,

March 30, I9I4. 